\title{
DICLORIDRATO DE BETAISTINA NA SÍNDROME VESTIBULAR PERIFÉRICA CANINA - Relato de caso
}

\author{
Alexandre Martini Brum, ${ }^{1}$ João Paulo da Exaltação Pascon, ${ }^{2}$ \\ Tatiana Champion ${ }^{3}$ e Mirela Tinucci-Costa ${ }^{4}$ \\ 1. Doutorando da FCAV-UNESP/Jaboticabal Docente da Universidade de Franca. E-mail: alexmbrum@bol.com.br \\ 2. Doutorando da FCAV-UNESP/Jaboticabal \\ 3. Doutoranda da FCAV-UNESP/Jaboticabal \\ 4. Docente do Departamento de Clínica e Cirurgia Veterinária FCAV-UNESP/Jaboticabal.
}

\section{RESUMO}

A síndrome vestibular periférica é uma condição clínica comum em cães. Várias doenças podem causar essa síndrome. Entretanto, sua patofisiologia ainda é pouco conhecida. As alterações clínicas geralmente são autolimitantes, a recuperação pode ser longa e, em casos crônicos, os déficits neurológicos podem ser irreversíveis. Em medicina veterinária, há poucas opções terapêuticas. $\mathrm{Na}$ Medicina, o dicloridrato de betaístina é amplamente utilizado. Essa medicação foi empregada em seis cães com síndrome vestibular periférica. Os resultados mostraram melhora clínica com sete a dez dias de tratamento e recuperação quase completa entre vinte e trinta dias. Este trabalho descreve a utilização da betaistina em cães com síndrome vestibular periférica, a rápida melhora clínica e ausência de efeitos adversos. Os resultados obtidos parecem justificar o uso de dicloridrato de betaistina na terapia de distúrbios vestibulares periféricos em animais de companhia.

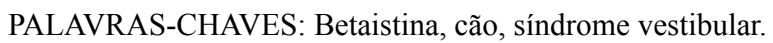

\section{BETAHISTINE DIHYDROCHLORIDE IN CANINE PERIPHERAL VESTIBULAR SYNDROME - Case report}

Vestibular disease is a common syndrome in small animals that may resulst of central or peripheral disease. The pathophysiology of peripheral vestibular syndrome is unknown, however it can be related to an abnormal dynamic of endolymphatic fluid or neuritis of the vestibular portion of the VIII cranial nerve. The recovery of neurological sings is slow and, in chronic cases, the neurological deficits can be irreversible. In veterinary medicine, thera are few medical options to treat this condition, however, in Medicine, betahistine dihydrochloride is used to treat peripheral vestibular disorders. These drug was used in four dogs with ves- tibular syndrome. The results showed clinical improvement in 7 to 10 days of treatment and completed recovery in 20 to 30 days, followed by the cure. One year after the treatment, the dogs did not have recurrence of the syndrome. This report shows the use of betahistine dihydrochloride in dogs with peripheral vestibular syndrome, with rapid clinical recover, without laboratorial abnormalities or recurrence of the clinical signs. The results encourage the use of betahistine dihydrochloride in the treatment of peripheral vestibular disorders in small animals.

KEY WORDS: Betahistine, dog, vestibular syndrome. 


\section{INTRODUÇÃO}

A síndrome vestibular é um distúrbio neurológico causada por disfunção no sistema vestibular. Esta condição é comum em cães e gatos. Pode se apresentar sob a forma periférica e central. A primeira forma envolve receptores periféricos no ouvido interno e a segunda, os núcleos ou tratos do tronco cerebral (TAYLOR, 2003). A otite média-interna é uma importante causa de síndrome vestibular periférica nos pacientes veterinários, provavelmente a mais comum. Outras etiologias, como a idiopática, congênita ou secundária a traumas, infecções e neoplasias, também podem desenvolver a síndrome (INZANA, 2003). Sua patofisiologia ainda é pouco conhecida e acredita-se que possa estar relacionada à dinâmica anormal da endolinfa ou neurites da porção vestibular do VIII nervo craniano (SCHUNK \& AVERILL, 1983).

A otite média-interna pode ser sequela de uma otite externa. Neste caso, a disfunção vestibular periférica pode ocorrer pela presença de agentes infecciosos que causam lesão diretamente no ouvido médio e interno, ou pela produção de toxinas, que provocam inflamação do labirinto (TAYLOR, 2003). Labirintite linfocítica é outra causa de síndrome vestibular periférica nos cães, porém sua etiologia também não está determinada (FORBES \& COOK, 1991). A doença vestibular canina geriátrica e a síndrome vestibular idiopática felina também são causas importantes de síndrome vestibular periférica em pequenos animais, entretanto sua etiologia é desconhecida (TAYLOR, 2003).

Os sinais clínicos dessa síndrome desenvolvemse agudamente e podem ser precedidos por vômitos e náuseas. Os animais podem se apresentar com inclinação de cabeça, nistagmo horizontal ou rotatório não posicional e conjugado, desorientação, paralisia facial, síndrome de Horner, queda e rolamentos (INZANA, 2004). A vertigem periférica é causada por uma geração desordenada de impulsos nervosos que afetam o aparelho vestibular (LORENZ \& KORNEGAY, 2003). Clinicamente, pode ser diferenciada da síndrome central. No quadro de origem central, ocorre nistagmo posicional e não conjugado, que pode ser tanto horizontal, vertical ou rotatório, inclinação da cabeça, ataxia simétrica, perda da propriocepção e alterações de estado mental. Sinais cerebelares podem estar presentes (TAYLOR, 2003).
O diagnóstico baseia-se nos sinais clínicos, achados de otoscopia, citologia de conduto auditivo externo ou de material obtido através de meringotomia, radiografia, tomografia computadorizada ou ressonância magnética do crânio. Muitas vezes os exames complementares não evidenciam nenhuma alteração, levando a um diagnóstico de doença idiopática ou geriátrica, todavia pequenas lesões podem não ser evidenciadas (BAYENS-SIMMONDS et al., 1997).

Não há tratamento específico para a desordem neurológica, apenas para a causa primária. A doença pode apresentar caráter autolimitante. $\mathrm{O}$ tempo de recuperação das alterações neurológicas é variável, entretanto, em casos crônicos, os déficits neurológicos podem ser irreversíveis (CHRISMAN, 1985). Os animais afetados podem compensar o déficit neurológico completamente ou permanecerem com leves alterações. Nestes casos, a compensação visual e tátil é muito importante e geralmente precede a recuperação do sistema vestibular, entretanto recidivas podem ocorrer (LEE, 1983). Em casos graves de vômitos, pode-se indicar o uso de antagonistas dos receptores histaminérgicos $\mathrm{H}_{1}$ (difenidramina) ou fármacos vestibulossedativos (meclizina) (TAYLOR, 2003).

A betaistina é uma medicação utilizada em humanos com distúrbio vestibular periférico e até o momento não há relatos de uso clínico em cães. Trata-se de uma molécula que possui afinidade com os receptores $\mathrm{H}_{3}$ e $\mathrm{H}_{1}$ da histamina, tendo ação antagonista e agonista, respectivamente. A betaistina também possui ação agonista sobre os receptores $\mathrm{H}_{2}$, entretanto sua ação é fraca em todos os tecidos (GATER et al., 1986). A dispepsia é uma reação adversa ocasional, mas a betaistina não aumenta a secreção ácida estomacal no homem (COCHRAN et al., 1974). Em cães, também não foram detectados aumentos significativos de secreção ácida durante tratamento com esta droga (CURWAIN et al., 1973).

Sua ação sobre receptores $\mathrm{H}_{1}$ nos vasos sanguíneos do ouvido interno promove vasodilatação e aumento da permeabilidade, evitando, assim, a expansão de volume e distensão do sistema hemolinfático. Entretanto, sua ação agonista é fraca. Os receptores $\mathrm{H}_{3}$ encontrados nas membranas pré-sinápticas dos neurônios histaminérgicos e de outros neurônios no cérebro modulam a liberação de histamina e de outros neurotransmissores. Acredita-se que a interação com 
os receptores $\mathrm{H}_{3}$ pode ser o principal mecanismo através do qual a betaistina exerça seus efeitos clínicos, os quais decorrem do aumento de fluxo sanguíneo no ouvido interno e da redução da atividade emissora dos núcleos vestibulares no cérebro (AFANASYEVA et al., 2003). Com a inibição dos receptores H3, a betaistina induz a um aumento na síntese e liberação de histamina no núcleo tuberomamilar, que, por sua vez, está conectado ao núcleo vestibular, desempenhando este papel fundamental na recuperação de lesões vestibulares (TIGHILET et al., 2005).

Acrescenta-se ainda o efeito da betaistina sobre a dinâmica da endolinfa na compensação do distúrbio vestibular periférico (TIGHILET et al., 2002; AFANASYEVA et al., 2003). Essa droga também aumenta o fluxo sanguíneo cerebral em outras partes do cérebro, sendo indicada para diversos distúrbios neurológicos de origem central em humanos (MEYER et al., 1974). Em cães anestesiados, a administração intravenosa de $0,44 \mathrm{mg} / \mathrm{kg}$ de betaistina aumentou em aproximadamente $200 \%$ o fluxo sanguíneo cerebral, porém o significado clínico não foi explorado (ANDERSON \& KUBICEK, 1971; SMITH \& MEYER, 1976).

Em estudos experimentais, a droga mostrou-se útil na resolução dos sinais vestibulares de felinos domésticos (TIGHILET et al., 1995). Felinos tratados com betaistina apresentaram rápida melhora dos distúrbios vestibulares induzidos (neurectomia vestibular unilateral) em comparação aos animais tratados com placebo (LACOUR, 2000).

Com base nos resultados em felinos com doença vestibular induzida, utilizou-se dicloridrato de betaistina em seis cães com síndrome vestibular periférica. A dose foi extrapolada do trabalho de ANDERSON \& KUBICEK (1971), no qual aproximadamente $0,5 \mathrm{mg} /$ $\mathrm{kg}$ aumentou o fluxo sanguíneo cerebral de forma significativa. Na ausência de sinais gastrintestinais nos primeiros três casos, essa dose foi aumentada para $1 \mathrm{mg}$ / $\mathrm{kg}$, para facilitar a administração da medicação.

\section{RELATO DE CASO}

Seis cães com síndrome vestibular periférica foram tratados com dicloridrato de betaistina. Antes de iniciar o tratamento, submeteram-se os pacientes a um exame físico geral e neurológico, para caracterizar a síndrome vestibular periférica. Após, foram submetidos à avaliação hematimétricas, otológicas e radiográficas para caracterizar a possível etiologia do distúrbio vestibular. Selecionaram-se pacientes com síndrome vestibular com diagnóstico presuntivo de otite média-interna, doença vestibular geriátrica e doença vestibular idiopática.

Primeiro caso: Inicialmente, foi atendido um Pastor Alemão, fêmea, de 13 anos, com histórico de incoordenação motora e perda de equilíbrio havia um mês e vômitos e sialorreia fazia dois dias. Ao exame físico, o animal apresentava inclinação da cabeça e andar em círculo para o lado direito, ataxia e nistagmo horizontal. A avaliação otoscópica evidenciou radiopacidade em membrana timpânica direita e ausência de alterações em condutos auditivos. Foram realizados exames hematimétricos (hemograma, alaninaaminotransferase, creatinina) e radiografia de crânio. A avaliação hematimétrica não revelou alterações, contudo a radiografia de crânio, para avaliação das bulas timpânicas, mostrou aumento de radiopacidade bilateral. As suspeitas clínicas foram otite médiainterna e doença vestibular canina geriátrica. Com base no histórico, em sinais clínicos e nos resultados dos exames, iniciou-se tratamento para otite média-interna com cefalexina $(30 \mathrm{mg} / \mathrm{Kg}$ a cada doze horas, por via oral) e dicloridrato de betaistina $(0,5 \mathrm{mg} / \mathrm{Kg}$ a cada doze horas, por via oral). Após 21 dias de tratamento, o animal já apresentava melhora clínica sem sinais de perda de equilíbrio, ataxia ou nistagmo, permanecendo apenas com discreta inclinação de cabeça. O paciente não apresentou episódios de êmese ou diminuição do apetite durante o tratamento. Não houve recidiva clínica após a interrupção do tratamento.

Segundo caso: Uma cadela sem raça definida, de cinco anos, apresentava rolamento para o lado direito, inclinação de cabeça para o mesmo lado, perda de equilíbrio, nistagmo horizontal e incoordenação motora. Realizaram-se hemograma e perfil bioquímico sérico (proteínas totais, albumina, alanina aminotransferase e creatinina), que não revelaram alterações. Avaliação otoscópica, citologia do conduto auditivo externo e exame radiográfico simples, para avaliação de bulas timpânicas, também não revelaram alterações dignas de nota. Baseando-se no histórico, em sinais clínicos, mesmo com ausência de sinais radiográficos, estabeleceu-se o diagnóstico de síndrome vestibular periférica idiopática. Instituiu-se terapia única com dicloridrato 
de betaistina (0,5 $\mathrm{mg} / \mathrm{Kg}$ a cada doze horas), obtendose melhora clínica evidente após dez dias de tratamento e, aos trinta dias, remissão completa dos sinais clínicos. Não houve relatos de alterações gastrintestinais durante o tratamento. Não ocorreu recidiva da síndrome neurológica após a suspensão do tratamento.

Terceiro caso: Um Pastor Belga, macho, com 13 anos, apresentava inclinação da cabeça para o lado esquerdo, andar em círculos e nistagmo com fase rápida ipsilateral e ataxia. Não mostrava alterações em otoscopia, assim como no hemograma e perfil bioquímico. Foi estabelecido diagnóstico de síndrome vestibular geriátrica, sendo instituída terapia com o dicloridrato de betaistina $(0,5 \mathrm{mg} / \mathrm{kg}$ a cada doze horas). No terceiro dia de tratamento, o cão já não apresentava andar em círculo e nistagmo, apenas permanecia com discreta inclinação da cabeça. No décimo dia, o cão não apresentava alteração clínica, sendo a medicação suspensa. O paciente não mostrou alterações gástricas na época do tratamento. Após a suspensão do tratamento, não houve recidiva do quadro neurológico.

Quarto caso: Um animal da raça Cocker Spaniel, fêmea, com 11 anos de idade, apresentava inclinação lateral da cabeça de início súbito havia aproximadamente dez dias. Não mostrava ataxia, nistagmo ou outros déficits neurológicos. Não se encontraram alterações na otoscopia, citologia do conduto auditivo externo e no exame radiográfico de bulas timpânicas. Com base nos achados clínicos, estabeleceu-se o diagnóstico de síndrome vestibular periférica idiopática. Administrou-se dicloridrato de betaistina na dose de $1 \mathrm{mg} / \mathrm{Kg}$ a cada doze horas. Após uma semana de terapia, o animal apresentou melhora significativa do quadro neurológico, sem apresentar alterações gastrintestinais. A medicação foi suspensa no décimo dia e o animal não apresentou recidiva.

Quinto caso: Um canino SRD, fêmea, com 15 anos, apresentava andar em círculos, ataxia, inclinação da cabeça e nistagmo rotatório fazia quatro dias. No otoscopia, evidenciou-se conduto auditivo com secreção ceruminosa abundante. Na citologia do conduto auditivo externo, revelou-se grande quantidade de formas leveduriformes, sugerindo malasseziose. Após a lavagem do conduto, a otoscopia foi realizada novamente e não se evidenciou nenhuma alteração. A avaliação hematimétrica não revelou nenhuma alteração significativa, assim como a radiografia de bulas timpânicas. Suspeitou-se de síndrome vestibular geriátrica. Administrou-se o dicloridrato de betaistina na dose de $0,5 \mathrm{mg} / \mathrm{Kg}$ a cada doze horas. Após cinco dias de terapia, o animal apresentou melhora significativa do quadro neurológico, sem apresentar alterações gastrintestinais. A medicação foi suspensa no décimo dia e o animal não apresentou recidiva.

Sexto caso: Um Fila Brasileiro, macho, com 4 anos, apresentava síndrome de Horner, inclinação da cabeça e ataxia fazia duas semanas. O exame otoscópico não revelou nenhuma alteração. Realizadas radiografias simples e contrastada (canulografia) das bulas timpânicas, nenhuma alteração foi evidenciada. Os exames hematológicos também estavam dentro da normalidade. Com base no resultados dos exames, suspeitou-se de síndrome vestibular idiopática. Foi prescrito dicloridrato de betaistina na dose de $0,5 \mathrm{mg} /$ $\mathrm{Kg}$ a cada doze horas. Com dez dias de terapia, o animal apresentou melhora significativa do quadro neurológico, sem evidenciar alterações gastrintestinais. A medicação foi suspensa após duas semanas e o animal não apresentou recidiva.

\section{RESULTADOS E DISCUSSÃO}

Os distúrbios vestibulares periféricos podem decorrer de fatores ou causas muitas vezes não diagnosticadas por meio de exames laboratoriais. Nesses casos, avaliações clínica geral, otológica e neurológica minuciosa e criteriosa são de extrema importância para um diagnóstico mais preciso. Entre os pacientes descritos neste trabalho, não foram evidenciadas alterações significativas em perfil hematimétrico e citológico do conduto auditivo. Somente o exame radiográfico de um dos cães apresentou alteração, que pode ser decorrente da enfermidade ou mesmo estar relacionada à senilidade do paciente. A utilização de dicloridrato de betaistina resultou em melhora significativa do quadro clínico, com um tempo de remissão curto. A duração de tratamento foi curta, variando entre dez dias e duas semanas. Neste período, a melhora neurológica já era evidente, independente da causa da síndrome vestibular periférica. A resolução da ataxia e nistagmo deu-se rapidamente, enquanto a inclinação da cabeça se resolveu mais tardiamente. Como os parâmetros hematológicos se encontravam dentro dos valores considerados normais, e as avaliações clínicas 
e laboratoriais não contraindicavam a utilização da medicação, o cloridrato de betaistina foi prescrito e nenhum efeito adverso ou indesejável, durante o período do tratamento, foi notado.

A ação sinérgica, entre a estimulação de receptores H1, e inibição de receptores $\mathrm{H} 3$ são benéficas em pacientes com distúrbios vestibulares. Além disso, a administração oral de betaistina aumenta o fluxo sanguíneo coclear (MEYER et al., 1974). Com o aumento do fluxo sanguíneo, ocorre uma regularização da dinâmica da endolinfa. Esse fenômeno está relacionado com a recuperação da função vestibular (TIGHILET et al., 1995). Além disso, a ação antagonista H3 aumenta a síntese e liberação de histamina em porções do sistema vestibular, que desempenham papel fundamental na recuperação de lesões vestibulares (TIGHILET et al., 2002). Dessa forma, a betaistina auxilia os mecanismos adaptativos envolvidos na compensação vestibular, tanto dinâmica como estática (TIGHILET et al., 2005). Em felinos, vários estudos comprovam a eficiência da betaistina em acelerar a compensação vestibular (TIGHILET et al., 1995; LACOUR, 2000; TIGHILET et al., 2002; TIGHILET et al., 2005).

Como há semelhanças entre os distúrbios vestibulares dos animais domésticos e de humanos, algumas terapias utilizadas em medicina podem ser extrapoladas para a medicina veterinária. Dessa forma, teoricamente, medicações como o dicloridrato de betaístina, utilizados no tratamento de doenças do labirinto em humanos, podem ser empregados em cães e gatos, com distúrbios vestibulares periféricos. No presente estudo, o dicloridrato de betaistina mostrou-se uma excelente opção para ser utilizada isoladamente, ou como adjuvante à terapia para a síndrome vestibular periférica em cães. Nos casos de síndrome vestibular periférica por otite média-interna, medidas terapêuticas para o tratamento da causa primária devem ser empregadas, mas medidas auxiliares para facilitar a compensação vestibular podem acelerar a recuperação das alterações neurológicas, trazendo conforto para o paciente e seu proprietário. A persistência de algum grau de inclinação de cabeça é uma consequência esperada também em humanos com distúrbios vestibulares tratados com a betaistina (TIGHILET et al., 2002; AFANASYEVA et al., 2003). Segundo CHRISMAN (1985), em alguns animais, a recuperação pode ser demorada e alguns déficits neurológicos podem permanecer necessitan- do de intervenção clínica. As causas de desordens vestibulares periféricas são muitas e, em animais idosos, no caso da doença senil, a recuperação pode ser espontânea (INZANA, 2004). Contudo, mesmo nos casos em que ocorre recuperação espontânea, o dicloridrato de betaistina pode ser empregado para acelerar a compensação vestibular, tornando a recuperação mais rápida, como evidenciaram LACOUR (2000) e TIGHILET et al. (2005) em felinos. Recidiva dos sinais clínicos, que pode ocorrer em alguns casos (TAYLOR, 2003), não foi registrada nos animais avaliados após um ano de acompanhamento.

Apesar do número pequeno de animais, não foram notadas reações adversas à medicação. Alterações gastrintestinais, relatadas ocasionalmente em humanos, não foram evidenciadas nesses pacientes. Estudos em um número maior de pacientes veterinários são necessários para assegurar a segurança dessa medicação, entretanto, na opinião dos autores, a medicação parece ser segura e eficiente.

\section{CONCLUSÕES}

O uso do dicloridrato de betaistina na terapia de distúrbios vestibulares periféricos em animais de companhia causou rápida remissão do quadro clínico. Não se evidenciaram alterações clínicas, recidivas e efeitos adversos nesses pacientes. Um ponto importante foi a satisfação do proprietário no que diz respeito a uma rápida resposta ao tratamento. $\mathrm{O}$ uso de dicloridrato de betaístina pode ser indicado como monoterapia ou terapia auxiliar, para o tratamento de pacientes com síndrome vestibular periférica, independente de sua etiologia.

\section{REFERÊNCIAS}

AFANASYEVA, S.A.; GORBACHEVA, F. E.; NATYAZHKINA,G. $M$. Isolated vertigo: pathogenesis and efficacy of betahistine (Betaserc). Journal of Neurology, v. 4. p. 12-16, 2003.

ANDERSON, W. D.; KUBICEK, W. G. Effects of betahistine HCI, nicotinic acid, and histamine on basilar blood flow in anesthetized dogs. Stroke, v. 2, p. 409-415, 1971.

BAYENS-SIMMONDS, J.; PURCELL, T. P.; NATION, N. P. Use of magnetic resonance imaging in the diagnosis of central vestibular disease. Canadian Vetetrinary Journal, v. 38, p. 38, 1997. 
CHRISMAN, C. L. Neurologia dos pequenos animais. São Paulo: Roca, 1985.

COCHRAN, K. M.; ALIAN, J. G.; RUSSELL, R. I. A study of the effect of betahistine on gastric acid secretion in man. Current Medical Research and Opinion, v. 2, n. 2, p. 6366,1974

CURWAIN, B. P.; HOLT, P.; SPENCER, J. The effect of betahistine on gastric acid secretion and mucosal blood flow in conscious dogs. British Journal of Pharmacology, v. 46, n. 2, p. 351-354, 1972.

FORBES, S.; COOK, J. R. Congenital peripheral vestibular disease attributed to lymphocytic labyrinthitis in two related litters of Doberman Pinscher pups. Journal of the American Veterinary Medical Association, v. 198, p. 447-449, 1991.

GATER, P. R.; WEBBER, S. C.; GUI, C. P. H.; JORDAN, C. C.; HAYES, M.A.; ASHFORD, J. J.; FOREMAN, J. C. Some studies of the action of betahistine at $\mathrm{H} 1$ and $\mathrm{H} 2$ receptors for histamine. Inflammation Research, v. 18, n. 3-4, p. 342-350, 1986.

INZANA, K. D. Distúrbios dos nervos periféricos. In: ETTINGER, S. J.; FELDMANN, E. C. Tratado de medicina interna veterinária. 5. ed. São Paulo: Manole, 2004. p. 699-722. v. 1.

INZANA, K. D. Síndromes vestibulares. In: PELLEGRINO, F. C.; SURANITI, A.; GARIBALDI, L. El libro de neurologia para la pratica clinica. Buenos Aires: Inter-médica, 2003, p. 239-244.

LACOUR, M. Vestibular compensation in the cat: the role of the histaminergic system. Acta Oto-Laryngologica, v. 120, p. 15-18, 2000.

LEE, M. Congenital vestibular disease in a German shepherd dog. Veterinay Records, v. 113, p. 571, 1983.
LORENZ, M. D.; KORNEGAY, J. N. Oliver \& Lorenz's handbook of veterinary neurology, Philadelphia: W. B.Saunders, 2003.

MEYER, J. S.; MATHEW, N. T.; HARTMANN, A.; RIVERA; V. M. Orally administered betahistine and regional cerebral blood flow in cerebrovascular disease. Journal of Clinical Pharmacology, v. 14 , p. 280-289, 1974.

SCHUNK, K. L.; AVERILL, D. R. Peripheral vestibular syndrome in the dog: a review of 83 cases. Journal of the American Veterinary Medical Association, v. 182, p. 1354-1357, 1983.

SMITH, K. A.; MEYER, M. W. Distribution of cardiac output in dogs during intravenous infusion of betahistine. Stroke, v. 7, p. 257-260, 1976.

TAYLOR, S. M. Head Tilt. In: NELSON, R. W., COUTO, C. G. Small animal internal medicine. 3. ed. St. Louis: Mosby, 2003. p. 1005-1009.

TIGHILET, B.; LEONARD, J.; LACOUR, M. Betahistine dihydrochloride treatment facilitates vestibular compensation in the cat. Journal of Veterinary Research, v. 5, p. 53-66, 1995.

TIGHILET, B.; TROTTIER, B.; LACOUR, M. Dose- and durationdependent effects of betahistine dihydrochloride treatment on histamine turnover in the cat. European Journal of Pharmacology, v. 523, p. 54-63, 2005.

TIGHILET, B; TROTTIER, S.; MOURRE, C.; CHOTARD, C.; LACOUR, M. Betahistine dihydrochloride interaction with the histaminergic system in the cat: neurochemical and molecular mechanisms. European Journal of Pharmacology, v. 20, p. 63-73, 2002.

Protocolado em: 25 set. 2007. Aceito em: 22 dez. 2009. 